\title{
Is the 8-mm robotic port safe? A case of trocar site hernia after robotic cholecystectomy using the da Vinci Xi system
}

\author{
Won Tae Cho, Tae Yoo, Sung Min Kim \\ Department of Surgery, Hallym University College of Medicine, Hwaseong-si, South Korea
}

Videosurgery Miniinv 2019; 14 (1): 137-140

DOI: https://doi.org/10.5114/wiitm.2018.76263

\begin{abstract}
With the increase in the frequency of robotic surgery, complications have also increased, including trocar site hernias, which rarely occur at the 8-mm port site after robotic cholecystectomy using the da Vinci Xi system. A 37-year-old woman was diagnosed with cholecystitis on abdominal sonography. She underwent robotic cholecystectomy using a bikini-line incision. However, after postoperative day 2, she presented to the emergency room with small bowel obstruction secondary to a herniated bowel loop through the left 8-mm port site. After failure to resolve the ileus, the patient underwent emergency surgery, and bowel resection and anastomosis of the ischemic area were performed. After surgery, the patient was discharged without complications. Although hernias develop less commonly at 8-mm robotic port sites, surgeons should carefully close the ports, especially in cases with large fascial defects or a high risk of herniation.
\end{abstract}

Key words: trocar site hernia, robotic cholecystectomy, bikini-line incision.

\section{Introduction}

Robotic surgical technology has revolutionized the field of minimally invasive surgery. Advantages of the commonly used da Vinci Xi system include a wider range of instrumental mobility, higher magnification, and improved 3-dimensional visualization, which have facilitated precise operating techniques in a variety of general surgery procedures. Moreover, with trocar incisions measuring less than $10 \mathrm{~mm}$, the diversity of trocar location and reduction in fascial torsion ensure less pain and better cosmesis, leading to patient satisfaction [1]. Therefore, robotic procedures have been used in most specialties of general surgery including robotic cholecystectomy.

However, as surgical cases have increased, complications have increased, including trocar site hernias (TSHs), which rarely occur at 8-mm port sites. To avoid TSH, the da Vinci Xi system includes a blade- less, blunt-tip 8-mm trocar that does not require fascial closure in non-midline port sites [2]. The main theoretical advantage of TSH reduction is that fascial and muscular layers are split and not cut on entry. We report a case of postoperative TSH following robotic cholecystectomy using the da Vinci Xi system.

\section{Case report}

A 37-year-old woman (informed consent was obtained) was diagnosed with calculous cholecystitis on abdominal sonography. There was no bile duct stricture or stone on magnetic resonance cholangiopancreatography. Her body mass index (BMI) was $30.2 \mathrm{~kg} / \mathrm{m}^{2}$, and she had no significant medical history except for 2 cesarean deliveries. She underwent robotic cholecystectomy with the bikini-line incision technique in which ports are placed as described in Figure 1 ( $8-\mathrm{mm}$ port at the right, middle, and left

\section{Address for correspondence}

Tae Yoo MD, Department of Surgery, Hallym University College of Medicine, 7 Keunjaebong-gil, 445-907 Hwaseong-si, South Korea, phone: 8201022984805 , e-mail: youts@hanmail.net 


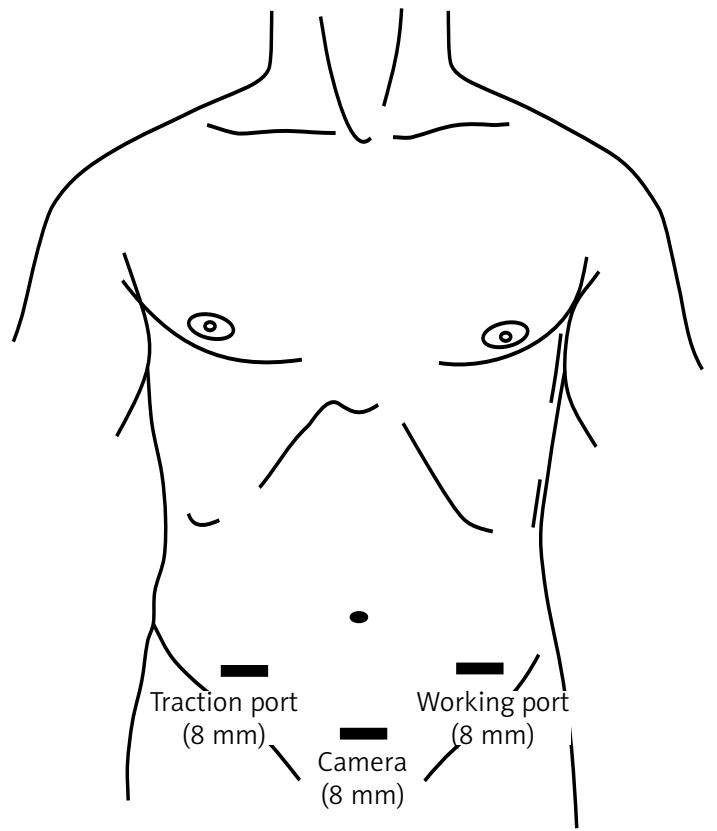

Figure 1. Three robotic cholecystectomy port sites. The $8-\mathrm{mm}$ trocars were inserted through the bikini-line incision (right, middle, and left lower quadrant abdominal areas with transverse incision)

lower quadrants) [1]. The operative time was $75 \mathrm{~min}$, with no drainage or intraoperative complications. After the specimen was retrieved from the mid-lower incision site, no fascial closure was performed. The patient was discharged 2 days postoperatively, after an uneventful recovery. Later on the day of discharge,

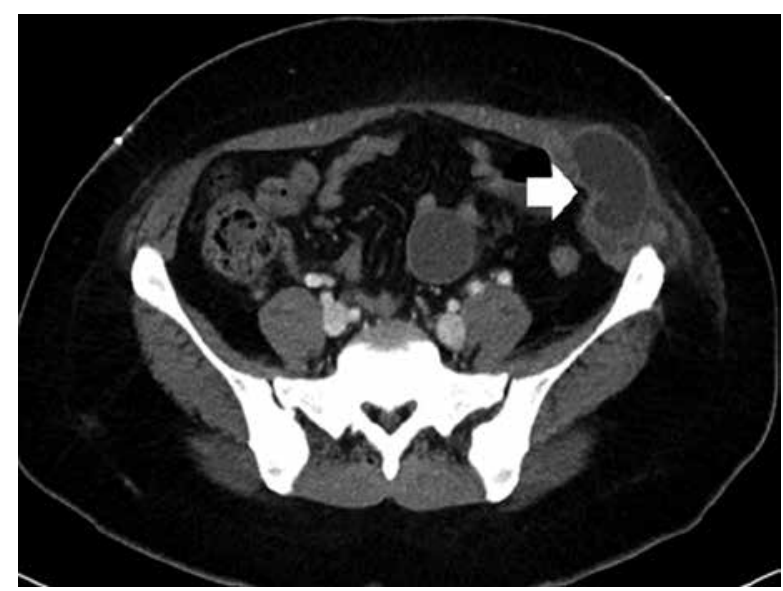

Photo 1. Arrow on the abdominal computed tomography image showing a loop of the small bowel herniating through a defect of fascial and peritoneal layers at the left $8-\mathrm{mm}$ port site the patient presented to the emergency room with complaints of left incision site pain, a distended abdomen, and 2 episodes of vomiting since discharge. Physical examination showed tenderness in the left lower quadrant with a $3-\mathrm{cm}$ palpable mass above the 8-mm port-site scar. There was no sign of peritonitis. All hematological and biochemical workups were within normal limits. Abdominal radiographs showed no sign of obstruction in the small and large bowel loops. Computed tomography of the abdomen revealed small bowel obstructions secondary to dilated fluid-filled herniated loops through abdominal fascial and peritoneal layers at the $8-\mathrm{mm}$ port site (Photo 1). After 5 days of conservative treatment and failure to resolve the mechanical ileus, the patient underwent emergency diagnostic laparoscopy. The herniated loop was twisted and obstructed within the fascial defect (Photo 2). The loop was delivered after the defective fascial and peritoneal layers were carefully dissected. Gangrenous changes were present at the part of the loop adherent to the defective area. The gangrenous part of the small bowel loop was kept under a warm saline-soaked sponge while the patient was given $100 \%$ oxygen for approximately $5 \mathrm{~min}$. However, the color and peristalsis of the gut loop did not significantly improve. Therefore, bowel resection and anastomosis of the ischemic area were performed through a small laparotomy incision near the umbilicus. The abdominal herniation site was closed in layers, allowing proper anatomical repair of the fascia and peritoneum. The patient remained in good condition during the postoperative period and was discharged without further complications.

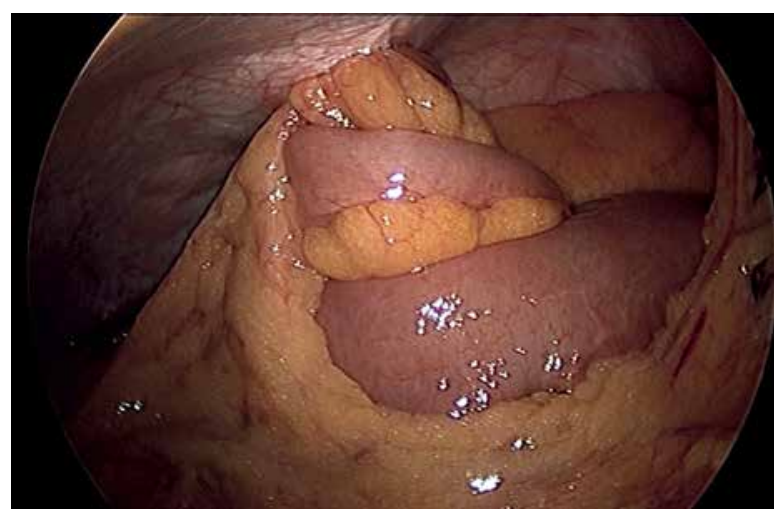

Photo 2. Laparoscopic picture showing a small bowel loop twisted and herniated through the left 8-mm port site 


\section{Discussion}

Trocar site hernia is defined as an incisional hernia at the site of trocar entry after laparoscopy. This is a well-known but rare complication after laparoscopic surgery, and may lead to serious adverse events, such as bowel incarceration and evisceration. The incidence of TSH after laparoscopic surgery has been estimated as 21 per 100,000 laparoscopic procedures, based on a survey of over 4 million American gynecologic laparoscopy procedures. Most (86.3\%) TSH cases occurred in ports $>10 \mathrm{~mm}$ in diameter, while only $2.7 \%$ occurred in ports $<8 \mathrm{~mm}$ in diameter in that study [3]. Bunting recently reported 99 (1.7\%) cases of TSH in 5,984 patients following laparoscopic cholecystectomy. Only $2 \mathrm{TSH}$ cases occurred through ports that were $5 \mathrm{~mm}$ in diameter, and the remainder occurred in ports $\geq 10 \mathrm{~mm}$ in diameter [4]. Clark et al. reported 3 cases of TSH among 500 patients following robotic gynecologic surgery, all at 12-mm trocar sites, without any herniations at 8-mm port sites [5]. There are few reports of TSH in general robotic surgery; hence, to the best of our knowledge, this is the first case of TSH after robotic cholecystectomy using the da Vinci Xi system.

Apart from the port size, several predisposing factors for TSH have been reported and categorized. Surgical factors include the sizes of trocars, use of cutting versus bladeless trocars, duration of the surgery, location of the ports, failure to surgically close port sites, excessive or inappropriate manipulation at the port sites, and trocar insertion or withdrawal techniques. Patient-related factors include obesity, increased abdominal pressure, poor nutrition, diabetes mellitus, chemotherapy, smoking, and wound infection.

At our institute, approximately 100 robotic cholecystectomies using the da Vinci system have been performed by a single surgeon, and this was the only case of TSH involving the $8-\mathrm{mm}$ port site at a non-midline incision. We believe that the TSH might have occurred for the following reasons. First, obesity may be a risk factor for TSH. The da Vinci Xi system uses pivoting point remote center technology. The robotic arm moves around a fixed "remote center" located on the fascial layer; thus, reducing trauma to this layer is an important factor for TSH. However, if the remote center of the robotic port is distant from the fascial layers, the robotic arm can damage the fascia around the port, resulting in an unintended larger defect. In patients with obesity, it is difficult for surgeons to detect the location of the fascial layer where the remote center should be fixed. Moreover, with pivoting action, the higher the $\mathrm{BMI}$ is, the larger is the peritoneal defect. Second, the working port itself may increase the risk of hernia. In this case, the herniation occurred on the left side (Photo 1) and involved the left working port. The robotic arm for the working port conducts more technically demanding procedures while the arm for gallbladder traction moves with the working port. This movement leads to further stretching and traumatization of the abdominal wall and fascial and peritoneal layers. Third, the prone position of the robotic arm may also have been a risk factor for TSH in our case. We adapted the robotic cholecystectomy with the bikini-line incision technique for cosmesis and pain relief [1]. However, all the port sites were far from the gallbladder and the robotic arms should have been properly laid down to reach the operative field. This position may lead to inappropriate location of the robotic port and cause a larger fascial defect.

Due to the rarity of TSH at laparoscopic or robotic trocar sites in non-midline incisions, routine fascial closure may not be necessary in all patients. Several studies have recommended that incision sites less than $10 \mathrm{~mm}$ long do not require fascial closure as in the case of non-midline sites [2]. Montz et al. also reported that most (76\%) of the TSH cases occurred near the midline trocar site [3]. However, we suggest that meticulous closure of the fascial defect may be needed in cases with large peritoneal and fascial defects or high-risk factors for TSH, even at 8-mm trocar or non-midline sites. Moreover, the trocar should be removed under direct vision and illumination to prevent any bowel entrapment or port removal and fascial defects at the port site $[6,7]$. After TSH occurred, we changed the working port placement instead of using the previous bikini-line incision. We placed a working port at the umbilicus due to the proximity to the fascial defect as well as for the cosmetic outcome without a visible scar.

\section{Conclusions}

Although TSH after robotic cholecystectomy using the da Vinci Xi system is uncommon, it may lead to serious complications such as bowel incarceration 
or evisceration. Furthermore, even though an 8-mm robotic port in a non-midline site has a lower likelihood of developing a TSH, surgeons should carefully close trocar sites, especially in cases with large fascial defects or higher risk of TSH.

\section{Conflict of interest}

The authors declare no conflict of interest.

\section{References}

1. Kim JH, Baek NH, Li G, et al. Robotic cholecystectomy with new port sites. World J Gastroenterol 2013; 19: 3077-82.

2. Liu CD, McFadden DW. Laparoscopic port sites do not require fascial closure when nonbladed trocars are used. Am Surg 2000; 66: 853-4.

3. Montz FJ, Holschneider CH, Munro MG. Incisional hernia following laparoscopy: a survey of the American Association of Gynecologic Laparoscopists. Obstet Gynecol 1994; 84: 881-4.

4. Bunting DM. Port-site hernia following laparoscopic cholecystectomy. JSLS 2010; 14: 490-7.

5. Clark LH, Soliman PT, Odetto D, et al. Incidence of trocar site herniation following robotic gynecologic surgery. Gynecol Oncol 2013; 131: 400-3.

6. Pesta W, Kurpiewski W, Luba M, et al. Single incision laparoscopic surgery transabdominal pre-peritoneal hernia repair case report. Videosurgery Miniinv 2012; 7: 137-9.

7. Venclauskas L, Jokubauskas M, Zilinskas J, et al. Long-term follow-up results of umbilical hernia repair. Videosurgery Miniinv 2017; 12: 350-6.

Received: 12.04.2018, accepted: 27.05.2018. 